\title{
Success Rate of Endovascular Intervention in the Treatment of Central Venous Stenosis or Occlusion in Hemodialysis Patients
}

\author{
Mohamed M. El Yamany ${ }^{*}$, Ahmed T. Mehanna, and Hatem H. Mohamed \\ Department of Surgery, Vascular and Endovascular Unit, Suez Canal University Hospital, Ismailia, Egypt
}

\begin{abstract}
Background: Central venous stenosis or occlusion (CVSO) is a major complication in hemodialysis patients that significantly affects morbidity and failure of the peripheral arteriovenous fistula (AVF). However, the best treatment is still unknown (surgical or endovascular). Endovascular treatment is a widely accepted option that includes percutaneous transluminal angioplasty (PTA) with or without stenting. The optimal endovascular treatment is still undetermined. Aim: To study the success rate and effect of endovascular intervention as angioplasty alone or with stenting, in patients with CVSO. As optimal endovascular treatment is still undetermined. Patients and Methods: this is uncontrolled clinical trial was conducted in Suez Canal University Hospital in the period between July 2017 to July 2019 and follow-up for 12 months. Thirty-four patients with compromised upper limb arteriovenous fistula due to CVSO were included to assess the patency rate of primary angioplasty with or without stenting. Any patients with (mediastinal lesion invading central veins, ipsilateral peripheral vein stenosis, or infected AVF) were excluded. Results: The mean age of studied patients was $53.06 \pm 8.49$ with a slightly larger number (19) of females (56\%). Pre-intervention, the innominate vein was the highest affected with lesion by $41 \%$. Occlusion was in 9 patients (26\%), while; 25 patients $(74 \%)$ had stenotic lesion. The success rate of primary patency was $55 \%$, follow-up of the successful cases at 3,6 , and 12 months with patency rate $91 \%, 77 \%$, and $59 \%$ respectively. There was no significant difference in the patency rate during one-year follow-up regarding the use of angioplasty alone or with stenting. Conclusion: percutaneous transluminal angioplasty with or without stenting according to our results is successful, safe, and effective in managing central venous lesions in compromised AVF in hemodialysis patients.
\end{abstract}

Keywords: Angioplasty, Central veins, hemodialysis.

\section{Introduction}

Central venous stenosis or occlusion (CVSO) is a major complication in hemodialysis patients causing significant morbidity and failure of the peripheral arteriovenous fistula (AVF). The prevalence of CVSO in hemodialysis patients is between 25 $40 \%^{(1)}$. There are many causes of this disease; however, the most common cause is the prolonged use of central veins as temporary access for hemodialysis and ipsilateral $A V F^{(2)}$. The affection of patients with CVSO is venous hypertension. This leads to 
significant upper extremity edema associated with pain, ulcer formation, and limb dysfunction. This might result in scarifying the vascular access and even ligation; the endpoint as a radical solution(3). Despite various available options for CVSO treatment including surgical and endovascular intervention, the best treatment is still unknown. Although endovascular treatment is widely accepted option that include percutaneous transluminal angioplasty (PTA) with or without stenting, the optimal endovascular treatment is still undetermined $(4,5)$. In this study we studied the success rate and effect of endovascular intervention, in patients with CVSO with compromised upper extremity AVF in our department.

\section{Patients and Methods}

\section{Study Setting}

Thirty-four patients were included in the current clinical trial realized in Suez Canal University Hospital (SCUH), Vascular and Endovascular Department, between July 2017 and July 2019. And follow-up was for 12 months. This study was approved by the local ethical committee, and a written informed consent was obtained from all study participants.

\section{Study Population}

Patients were randomly selected from those who presented to our department through emergency or outpatients clinic suffering from 'compromised AVF due to CVSO and associated with symptomatic ipsilateral upper extremity edema with pain` were included in the study. Patients with any of the following were excluded from the study; abnormal uncorrectable bleeding profile, mediastinal lesions invading central veins, cardiac pacemaker, Ipsilateral peripheral vein stenosis, infected vascular access site, or lower limb vascular access.

\section{Procedures and Methods}

As pre-operative assessment, detailed history, examination, investigations, and imaging were performed to confirm the diagnosis and exclude patients with exclusion criteria. All patients were examined with duplex ultrasound, to assess patency of limb, neck veins and determining access site. Venography was used to assess the exact location, length of the lesion, and to know the type of tools to be used in the treatment.

\section{Anesthesia}

The procedures were performed under local anesthesia ( $2 \%$ lidocaine) with assistant of preoperative intravenous analgesics (meperidine or fentanyl) and backup general anesthesia was used in uncooperative patients $^{(6)}$.

\section{Procedure}

Patency of femoral and jugular veins was checked by duplex ultrasound before endovascular intervention in all patients. Patients were monitored by electrocardiogram and pulse oximeter. Prophylactic antibiotic (Cefotaxime, 2 g IV) was administered. Digital Venography was done to know the exact location, length, and severity of $\operatorname{CVSO}^{(6,7)}$. The puncture site was achieved by ante grade puncture of the efferent vein above the elbow and away $5 \mathrm{~cm}$ from the (AVF) anastomosis. After puncture and insertion of the introducing $6 \mathrm{~F}$ to $10 \mathrm{~F}$ sheaths, venography of central veins was done followed by probing of the steno-obstruction with a $4 \mathrm{~F}$ or $5 \mathrm{~F}$ Angelshaped catheter and a hydrophilic guide wire; (0.035 Terumo, Tokyo, Japan). If the lesion was passed through easily, the guide wire was changed with a stiff wire and a 10 or $16 \mathrm{~mm}$ percutaneous` angioplasty PTA 
was subsequently performed. If the lesion was tight and could not be crossed from this approach, the right internal jugular vein was tried as a substitute access ${ }^{(6)}$. In case of use left or right femoral catheterization as another approach, it was done under ultrasound guidance. An $8 \mathrm{~F}$ introducer sheath was used to deploy a $5 \mathrm{~F}$ Glide-catheter; (Terumo, Tokyo, Japan) to cross the CVSO and was captured from above with a 10-15mm snare (pull through technique). The used balloons (diameter: $6-16 \mathrm{~mm}$ ) were inserted through the AVF puncture site and were inflated over the stenotic or obstructed segments with high pressure (10-15 atm). Fortunately, this was achieved by two or three inflations of 2-3 min for each one. When the balloon was not fully expanded even with high pressure, two or three more dilatations were tried, in our study we used (Boston scientific, Mustang ${ }^{T M}$ PTA high pressure XXL Balloon Dilatation (atheter) ${ }^{(7)}$.

\section{Placement of stent}

The indications for stent insertion were $\geq 30 \%$ residual stenosis or instant recoil and persistent presence of collateral vessels after the angioplasty procedure. In our study we used self-expandable stents; (Boston scientific wall-stent in different diameters). Stents were chosen to match the characteristics of the lesion and the anatomical site. The length of the stent was determined to be 20mm longer than the lesion and to be dilated $10-20 \%$ larger than the diameter of the non-affected adjacent vein(7). Patients were prescribed Low Molecular Weight Heparin for 1 week after the procedure in therapeutic dose of $1 \mathrm{mg} / \mathrm{kg} /$ dose twice daily, and aspirin indefinitely. All patients treated with stents received a clopidogrelloading dose of $300 \mathrm{mg}$ and continued dual anti-platelet therapy for 2 months ${ }^{(8)}$.

Follow-up Examination Parameters Follow-up was started immediately to as sess the complications of the procedure as perforations, bleeding, puncture site hematoma. During later follow-up, the puncture site infection or pseudoaneurysm was assessed. During the regular follow-up visits, the evaluation of patency rate through clinical and radiological parameter was done. The clinical was the re-use of AVF efficiently, presence of collateral veins in the chest was and the affected arm, the resolution of the affected extremity edema and healing of skin macerations. The radiological assessment was not done to all patients as a routine through duplex ultrasound, only in case of suspected technical failure as the clinical parameters from the fistula functions and edema of the extremity. Primary patency of the technique was defined as a patent central vein without recurrent stenosis or occlusion and no need for further intervention within the central veins $^{(9)}$. Technical failure was defined as a $<50 \%$ gain in luminal diameter. Early failure was defined as an inability to cross the lesion at the time of the primary procedure or by the presence of an occlusion or $50 \%$ or more restenosis within the first 30 days after the initial procedure. Residual stenosis was defined as $\geq 30 \%$ remaining stenosis at the conclusion of intervention in comparison to adjacent, non-diseased vein ${ }^{(9)}$. All patients were followed at the outpatient clinic at 3, 6 months and 12 months after the procedure. Patients were regularly monitored during hemodialysis access by nephrologists and the hemodialysis team. If there were any symptoms or signs of restenosis of the central veins or if the dynamic venous pressures exceeded threshold levels during hemodialysis, the patients were referred to our vascular department. The patients with failed technique were shifted to another policy of management, as surgical bypass, or vascular access ligation, according to the anatomical site of lesion and patient's fitness for surgery. 


\section{Statistical Analysis}

Data were analyzed using statistical package for the social sciences, version 20 (IBM Corp, Released 2011, IBMSPSS Statistics for Windows, Version 20.0, Armonk, NY: IBM Corp.). Nonparametric Kruskal-Wallis and Mann-Whitney $U$ tests were used for analysis. Likert score averages were given as mean $\pm S D$. $P$ value less than or equal to 0.05 was set as a criterion for establishing statistical significance.

\section{Results}

Thirty-four patients with end stage renal disease (ESRD) were included in this study from those who presented to Vascular and Endovascular Department in SCUH, suffering from upper extremity edema related to their vascular access of hemodialysis, which resulted from CVSO, all patients underwent PTA alone or with selective stenting for the previously mentioned causes. The mean age of the studied patients was $53.06 \pm 8.49$ with slightly larger number of females to males, 19 (56\%) and 15 (44\%) respectively. The mean \pm SD period from AVF creation till the appearance of symptoms was $42.09 \pm 33.76$ months with range from 3 to 124 months. Regarding co-morbidities, nearly two third of the studied patients were hypertension 21 (62\%) while diabetes and smoking were presented in $13(38 \%)$ and $12(35 \%)$ respectively. Thirty-two patients (94\%) presented with arm swelling, while face oedema was present in $21 \%$ of cases. $29 \%$ of cases had elevated venous pressure during haemodialysis sessions $(\geq 150 \mathrm{mmHg}$, with an arterial blood flow of $230 \mathrm{ml} / \mathrm{min}$ ), prolonged bleeding was present in $24 \%$ of cases. Due to the mentioned presentations, inadequate dialysis with machine interruption was reported in $38 \%$ of cases, (figure 1 ).

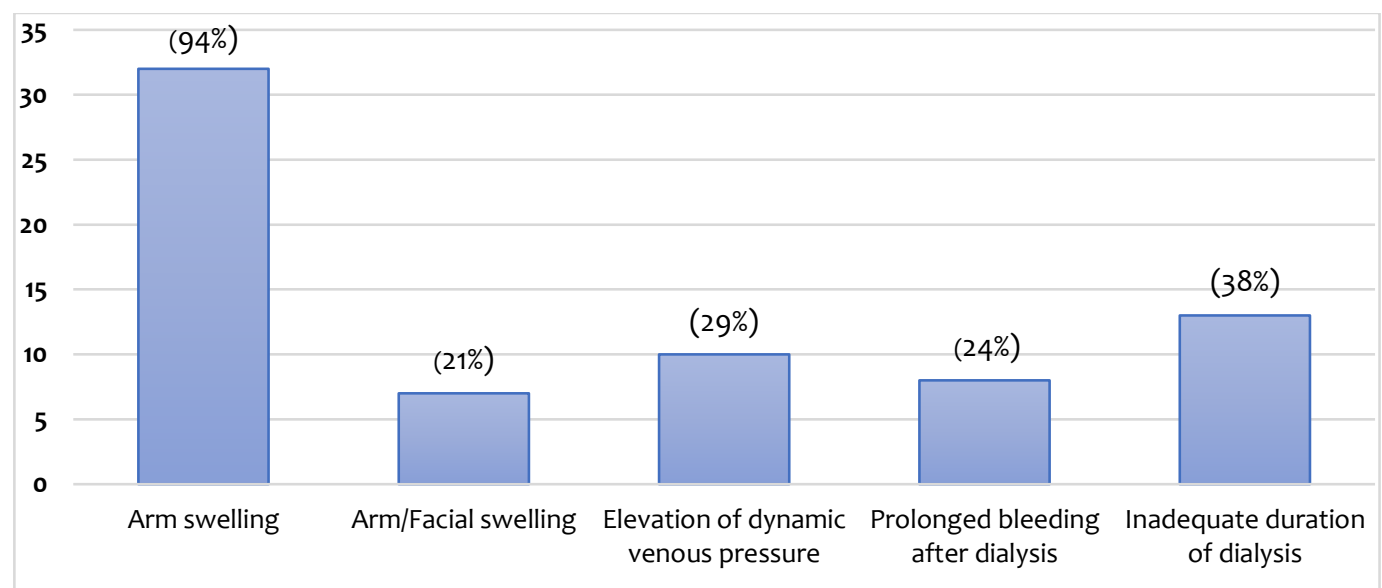

Figure 1: Patient presentation (Indication for intervention)

Native vascular access was seen in $94 \%$ of the cases while only 2 patients had synthetic access. The brachiocephalic access was the most common site of AVF in our patients (47\%) (table 1). Most of the patients underwent the procedure under local anesthesia 21(62\%). Ten patients (29\%) need additional sedation with local anesthesia, while only 3 patients (9\%) needed general anesthesia (orthopnea, back pain, or uncooperative patient) during the procedure. The access site of puncture was the dialysis vein in 28 patients (82\%), through combined access, femoral and the dialysis vein in 4 patients and 2 patients $(6 \%)$ through graft puncture. 


\begin{tabular}{|l|c|}
\hline \multicolumn{2}{|l|}{ Table 1: Characteristics of the arteriovenous fistula (AVF) } \\
\hline Characteristics of the (AVF) & $\mathrm{n}(\%)(\mathrm{n}=34)$ \\
\hline Type of AVF & $32(94 \%)$ \\
- Native & $2(6 \%)$ \\
- Synthetic & \\
\hline Site of AVF & $16(47 \%)$ \\
- Brachiocephalic & $11(32 \%)$ \\
- Brachio-basilic transposition & $5(15 \%)$ \\
- Radio cephalic & $2(6 \%)$ \\
- Brachio-axillary Graft & \\
\hline
\end{tabular}

Regarding the type of lesion in the central veins, stenosis was in seen 25 patients (74\%), and occlusion was seen in the rest of patients, as shown in figure 2 . Innominate vein lesions were presented in 14 patients (41\%), while superior vena cava (SVC) lesions were in only 4 patients (12\%), as shown in figure 3.

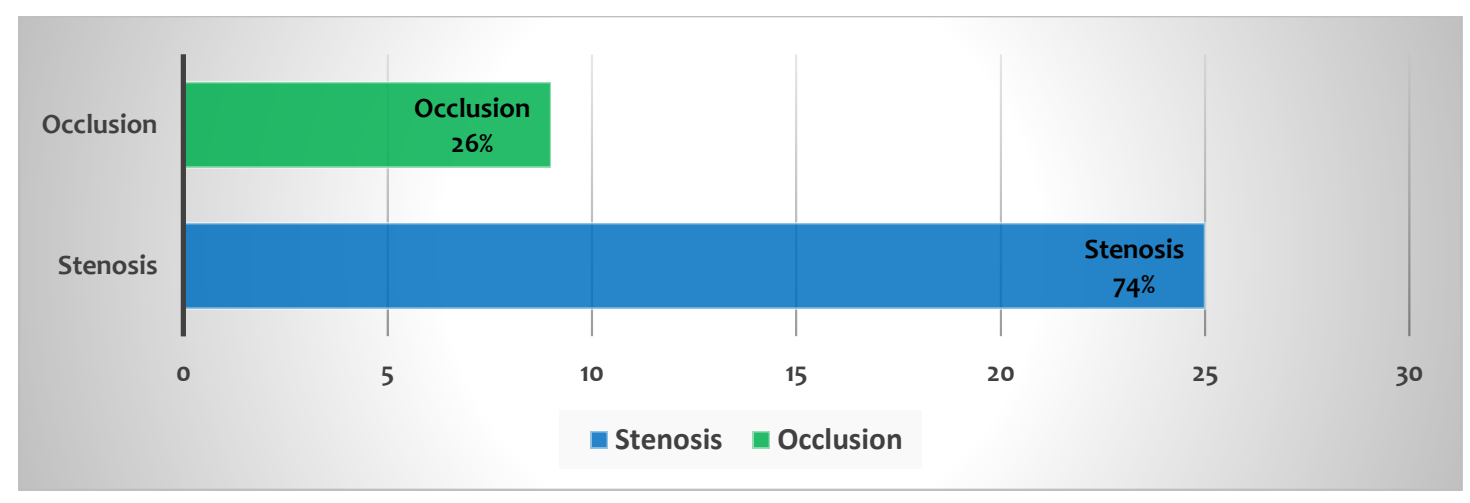

Figure 2: Distribution of the types of lesions

In our study, the technique was successful in 22 patients (65\%) during the one-year follow-up period and the rest was failed, as shown in figure 4 . The patients with failed technique were shifted to another policy of management as surgical bypass or ligation of the vascular access regarding the anatomical site of lesion and patient fitness for surgery. The technique was successful in 22 cases (65\%), 9 of them were in the innominate vein, stenting was needed in 5 patients (23\%) and the rest (18\%), angioplasty was enough to obtain targeted patency. Two patient had superior vena cava, both needed stenting as angioplasty was not enough, while in subclavian vein lesion, balloon dilation was enough in 5 patients, while 2 patients needed stenting, as shown in table 2. Unsuccessful cases were in 12 patients, most of them (92\%) were native vascular access, and innominate vein lesions were in 5 patients (42\%), while only 2 patients (17\%) were in axillary vein lesions. Stenosis was in (58\%) of unsuccessful cases while the rest was in occlusive lesions, as shown in table 3. 


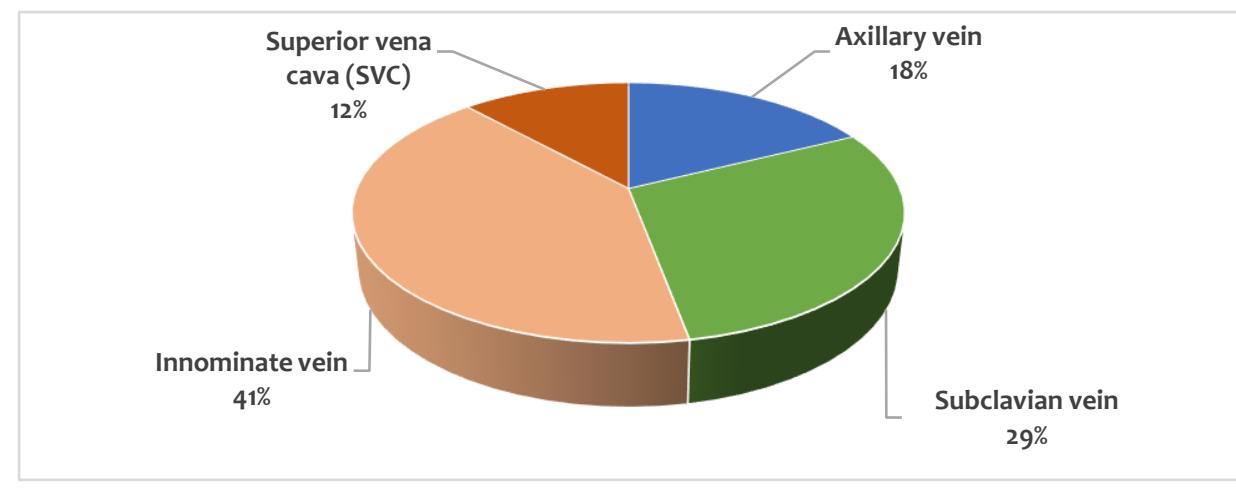

Figure 3: Anatomical Sites of lesions

After one-year follow-up, 13 patients (59\%) had successful patency rate, 5 patients (39\%) were in the innominate vein lesions, while 4 patients (45\%) had recurrence in the lesion were also in innominate vein. Regarding axillary vein lesions, success was in 3 patients (23\%) while recurrence was only in 1 patient. Majority of the successful cases was in single site lesion as 10 patients (77\%), while failure was high as in 7 patients (78\%) in multiple site lesions, as shown in table 3. Majority of successful cases after one-year follow-up (92\%) were in stenotic lesions, while (56\%) of recurrent cases were in occlusive lesions, after the same period of follow-up. All patients with successful patency were had native vascular access, as shown in table 4 . In the patent group after one-year follow-up, 7 patients (54\%) were had stent and the rest had only ballooning, while in the recurrent group after the same period of follow-up, 3 patients (33\%) and 6 patients (67\%) were had ballooning only, as shown in table 5 .

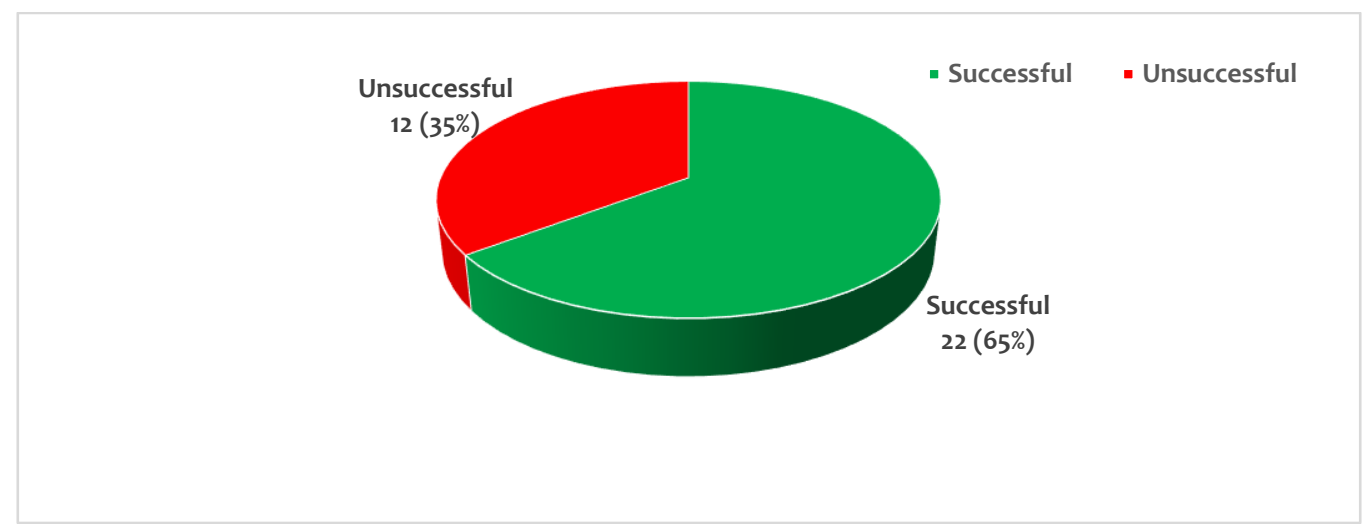

Figure 4: The outcome of the procedure after one-year follow-up

Regarding the complications of the technique, the incidence of post puncture hematoma was in 5 patients (15\%), while infection at the vascular site post-intervention was in 2 patients (6\%), these 7 patients were treated conservatively with proper dressing, while in 27 patients (79\%) went without any complications recorded. During the follow-up period, the patency rate was $91 \%$ of cases after 3 months of followup, then after 6 months from the procedure, the patency rate was dropped to $77 \%$, and after 12 months the patency became 59\% (Figure 5). 
Table 2: Distribution of the Successful cases (Balloon angioplasty with or without stenting classified by the sites of lesions)

\begin{tabular}{|l|c|c|c|}
\hline \multirow{2}{*}{ Site of lesions } & Non-stenting group & Stenting group & \multirow{2}{*}{ P value } \\
\cline { 2 - 3 } & $\mathrm{n}(\%)$ & $\mathrm{n}(\%)$ & \\
\hline Axillary vein & $3(14 \%)$ & $1(4 \%)$ & 0.46 \\
\hline Subclavian vein & $5(23 \%)$ & $2(9 \%)$ & 0.40 \\
\hline Innominate vein & $4(18 \%)$ & $5(23 \%)$ & 0.23 \\
\hline SVC & 0 & $2(9 \%)$ & 0.81 \\
\hline Total & $14(55 \%)$ & $10(45 \%)$ & \\
\hline
\end{tabular}

\begin{tabular}{|c|c|}
\hline According to & \\
\hline \multicolumn{2}{|l|}{ Type of AVF } \\
\hline - Native & $11(92 \%)$ \\
\hline - Synthetic & $1(8 \%)$ \\
\hline \multicolumn{2}{|l|}{ Type of lesion } \\
\hline - Stenosis & $7(58 \%)$ \\
\hline - Occlusion & $5(42 \%)$ \\
\hline \multicolumn{2}{|l|}{ Site of lesion } \\
\hline - Axillary vein & $2(17 \%)$ \\
\hline - Subclavian vein & $3(24 \%)$ \\
\hline - Innominate vein & $5(42 \%)$ \\
\hline - Superior vena cava (SVC) & $2(17 \%)$ \\
\hline
\end{tabular}

\section{Discussion}

Obstruction or stenosis of the central veins are a major concern in patients undergoing prolonged hemodialysis causing obvious morbidity with dysfunction of the access site. CVSO potentially affects the patency by diminishing flow or leading to venous hypertension and lead to edema of the upper extremity which necessitating access ligation to subside the complications and relief of symptoms ${ }^{(10)}$. The major risk factor for the development of CVSO is previous prolonged history of central venous catheter where it acccounts for $27 \%$ of cases with specifically higher incidence (42\%) if placed as subclavian access compared with a $10 \%$ rate with catheters placed in internal jugular vein access ${ }^{(11,12)}$.

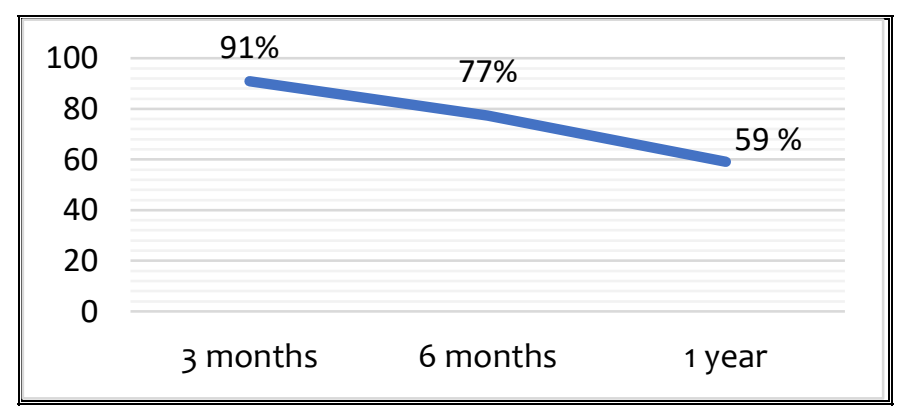

Figure 5: Patency rate of the successful cases through one-year visits of follow-up 


\begin{tabular}{|c|c|c|}
\hline \multirow{2}{*}{$\begin{array}{c}\text { Table 4: One-year follow-up regarding the patency rate of successful } \\
(n=22) \text { cases according to the procedural variables }\end{array}$} \\
\hline \multirow{2}{*}{ Site of the lesions } & \multicolumn{2}{|c|}{$n(\%)$} \\
\cline { 2 - 3 } & $\begin{array}{c}\text { Patent }(n=13) \\
59 \%\end{array}$ & $\begin{array}{c}\text { Recurrent }(n=9) \\
41 \%\end{array}$ \\
\hline- Axillary vein & $3(23 \%)$ & $1(11 \%)$ \\
\hline- Subclavian vein & $4(31 \%)$ & $3(33 \%)$ \\
\hline- Innominate vein & $5(39 \%)$ & $4(45 \%)$ \\
\hline- Superior vena cava (SVC) & $1(8 \%)$ & $1(11 \%)$ \\
\hline
\end{tabular}

\begin{tabular}{|c|c|c|}
\hline \multicolumn{2}{|c|}{ Table 5: One-year follow-up patency rate according to } \\
AVF characteristics $(\mathrm{n}=22)$
\end{tabular}

The objective of this study was to assess our experience with PTA for symptomatic lesions and to measure the success rate and the effectiveness of this approach for improving patient's symptoms and maintaining AVF patency. The primary objective of the study was to assess the success rate, efficacy, and safety of percutaneous angioplasty of central venous lesions in hemodialysis patients presented to the Vascular unit of SCUH. Thirty-four patients were included in our study with ESRD, with mean age of $53.06 \pm 8.94$ years, having upper limb AVF presented with venous hypertension. Mean age was lower than Sprouse Il et al ${ }^{(8)}$, and Shi et al(13), representing 67.2 and 66.4 respectively, still more than Yadav et al. ${ }^{(14)}$, with a mean of 46 years.

Table 6: The patency rate of the stented cases after one-year follow-up $(n=22)$

\begin{tabular}{|l|c|c|}
\hline \multirow{2}{*}{ Type of intervention } & \multicolumn{2}{|c|}{$\mathrm{n}(\%)$} \\
\cline { 2 - 3 } & Patent $(n=13)$ & Recurrent $(n=9)$ \\
\hline Stenting & $7(54 \%)$ & $3(33 \%)$ \\
\hline Non-stenting & $6(46 \%)$ & $6(67 \%)$ \\
\hline
\end{tabular}



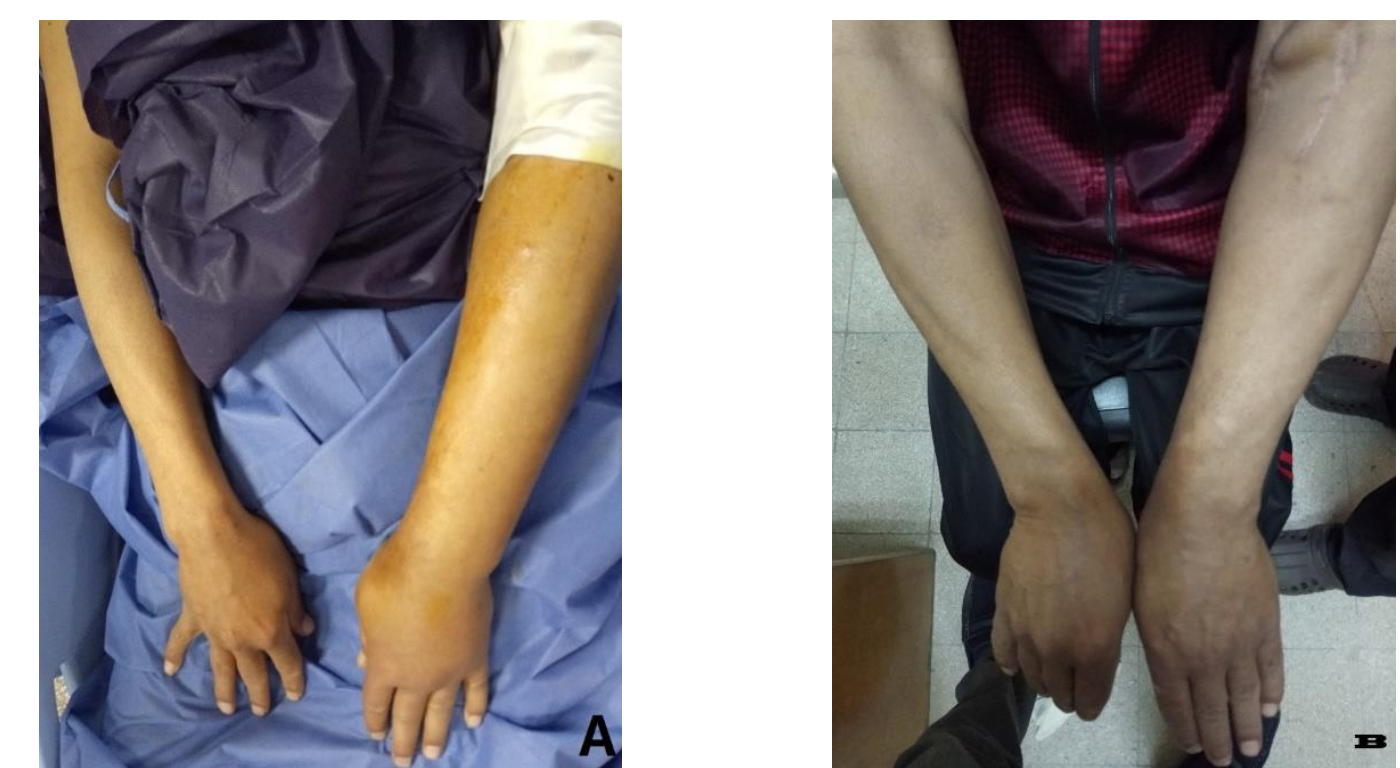

Image 1: image (A) shows 48 years old male patient known to have ESRD with 10 presented with left upper limb swelling and pain. Native brachiocephalic fistula is present in the limb with appearance of symptoms and signs 5 months after fistula creation, image (B) shows the patient after PTA 3 months.

We found that $62 \%$ of our patients were hypertensive and $38 \%$ were diabetic. This was in concordance with Surowiec et al. ${ }^{(10)}$, who reported that $60 \%$ of their patients were hypertensive and $48 \%$ were diabetic. This was also in line with Nael et al. ${ }^{(16)}$, who found that $48 \%$ of their patients were hypertensive, $45 \%$ of patients were diabetics, and $45 \%$ of patients had significant coronary artery disease. The mean \pm SD period from AVF creation till the appearance of symptoms was $42.09 \pm 33.76$ months with a range from 3 to 124 months, this mean was much longer than Yadav et $\mathrm{al}^{(14)}$, and Atalla et $\mathrm{al}^{(2)}$, with means of 36 and 20 months respectively. This could be explained by the shorter period of stay with neck catheter in the target population which decreases both incidence and degree of stenosis, this was still lower than Christidou et al. (17), with mean \pm SD of $69 \pm 44.6$. Most of our patients (94\%) complained of obvoius arm swelling while addiotional ipsilateral facial swelling was reported in $21 \%$ of cases. This was in concomitant with Christidou et al. ${ }^{(17)}$, who reported arm swelling in $73 \%$, facial swelling in $18 \%$ of studied population and
Kalman et al. ${ }^{(18)}$, whose study showed arm swelling in $80 \%$ of patients. Failure to obtian a venous access and Inadequate dialysis was reported in $38 \%$ of study population, more than those $(8 \%)$ reported by Kalman et al. ${ }^{(18)}$. We found that $58.82 \%$ of lesions were left sided, this was in line with Christidou et al. (17), and Atalla et al.(2), where left sided lesions were $68.18 \%$ and 63.6 respectively. This could be explained that the non dominant limb (left) is selected more than the dominant (right) limb. In our study, $47 \%$ of the patients had brachiocephalic AVFs, which is in line with Oguzkurt et al.(15), Nael et al.(16), and Atalla et al. (2), who found that most of patientshad brachiocephalic AVF, representing $66 \%, 69.1 \%$ and $44 \%$ respectively. Lesions were accessed through the dialysis vein in $82 \%$ of cases, while femoral veous access was recorded in $12 \%$ of cases, this is in line with Christidou et al. ${ }^{(17)}$, who also share the same percent (82\%) in accessing dialysis vein while femoral vein was accessed in $9 \%$ of cases. patients followed by the subclavian vein in $29 \%$ of patients, the axillary vein in $18 \%$, and superior vena cava in four cases (12\%). This 
was in agreement with Shi et al. ${ }^{(13)}$, and Yadav et al. ${ }^{(14)}$, who stated that most lesions were located at innominate vein, representing $91.6 \%$ and $72.7 \%$, respectively. However, Young et al.(19), Surowiec et al.(10), and Bakken et al.(9), reported that most lesions were located at subclavian vein, representing $48.6 \%, 67.5 \%$ and $48 \%$ of their lesions, respectively. In our study, we found that $74 \%$ of lesions were stenosis, and the remaining $26 \%$ were occlusive in nature, which is in agreement with Young et al.(19), Aytekin et al. (20), and Atalla et al. ${ }^{(2)}$, who found that most lesions were stenosis, representing $79.2 \%, 78.5 \%$ and $80 \%$ respectively.
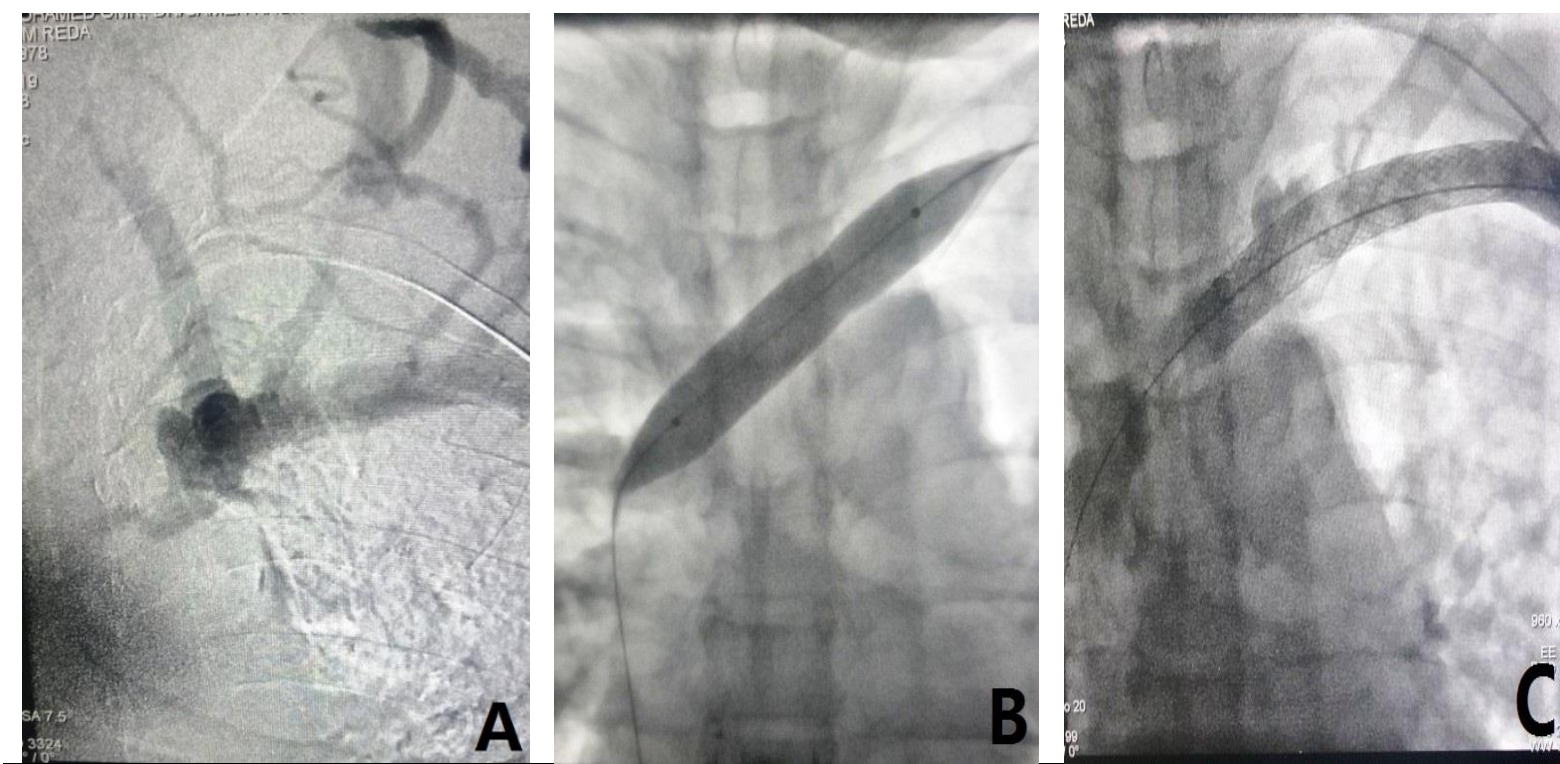

Image 2: These three images show the different stages of management of left innominate occlusion, image (A) shows the cut-off of the contrast at the origin of the left innominate. Image (B) show the balloon angioplasty of the occlusion after intra-luminal wire crossing. Image (C) stenting after balloon dilatation due to residual stenosis more than $30 \%$ of the vein lumen.

In the our study, the lesions were most commonly located in the innominate vein in $41 \%$ of However, Dammers et al. (3), Shi et al.(13), and Yadav et al. ${ }^{(14)}$, reported that central venous occlusion was seen in $60.7 \%, 58.3 \%$, and $61.1 \%$, respectively. In this study, initial percutaneous angioplasty wastechnically successful in $65 \%$ of cases, keeping with Surowiec et al.(10), Shi et al. ${ }^{(13)}$, and Yadav et al. ${ }^{(14)}$, who reported that technical success rate was $89 \%, 83.3 \%$, and $81.8 \%$, respectively. Yadav et al. ${ }^{(14)}$, included 11 patients, in which technical success was achieved in $81.8 \%$ cases (9/11) while the remaining two patients experienced occluded segments that could not be negotiated, giving total number of nine patients in whom the procedure was successful. In our study, $45 \%$ of the lesions had primary stenting, which is midway between Sprouse II et al. ${ }^{(8)}$, where $19 \%$ patients had stent and a study performed by Shi et al.(13), who reported that $55 \%$ of cases had primary stenting. Furthermore, Yadav et al. ${ }^{(14)}$ reported that PTA alone was done in 2 patients (22.3\%) while, 7 patients (77.7\%) had balloon angioplasty with stenting. In our study, one-year patency rate for stented cases was $62 \%$ and $38 \%$ for cases with PTA alone, which was statistically insignificant. This is in contrast to Christidou et al. ${ }^{(17)}$, who stated that the $3,6,12$, and 24 month primary patency rates were $88.3 \%, 65.3 \%, 45.6 \%$, and $25.5 \%$, respectively. This was in lines with Shi et al. ${ }^{(13)}$, where the primary patency rates were $48.6 \pm 18.7 \%$ in the PTA group alone, and $77.1 \pm 14.4 \%$ at one-year after treatment 
in the PTA with stent group. These high rates for stent group can be explained as PTA was performed in 9 patients and stenting was performed in 11 patients, where as in our study, the number of stented cases was 12 in 22 successful cases. Moreover, the patency rates of the 22 patients with susscessful intervention collectively in this study were $91 \%, 77 \%$, and $59 \%$ at 3, 6, and 12 months, respectively. However Shi et al. ${ }^{(13)}$, found that the over all primary patency rates of 22 patients in whom 11 patients had stenting were $88.9 \pm 10.5,64.8 \pm 10.5$, and $48.6 \pm 18.7 \%$ at 3,6 months, and one-year post-operatively in the PTA group and $90.0 \pm 9.5 \%$ and $77.1 \pm 14.4 \%$ at 6 months and one-year postoperatively in the stent group, respectively. In our study, there were minor procedure-related complications at the access site. Fifteen percent of cases had puncture site hematomas while only (6\%) had puncture site infection. One case of peri-procedural mortality (3 days postprocedure) at distant hospital with no clear explanation of the cause of death. While Sprouse et al. ${ }^{(8)}$, documented no complications, Nael et al. ${ }^{(16)}$ observed two complications that required further intervention. One-patient with right axillary and subclavian veno-occlusive disease developed a pseudoaneurysm subsequent to PTA that was successfully treated with a covered stent. In onepatient with complete occlusion of the right subclavian and brachiocephalic veins, the angioplasty balloon $(8 \mathrm{~mm})$ ruptured at 20 atm. Many attempts to retrieve the ruptured balloon failed because the ruptured balloon catheter could not be pulled out through the previously inserted Smart stent in the right axillary vein. This patient was taken to surgery, and the ruptured balloon was retrieved with a direct cut down of the axillary vein. Dammers et al.(3), documented few complications occurred in six patients resulting in dissection, wall-stent dislocation, and limited contrast extravasate. The dissection was successfully treated by stent implantation. Stent dislocation was solved by the insertion of an overlapping anchoring stent. In 3 of 4 patients with an extravasate during PTA, angioplasty was still successful. Recanalisation of an occluded subclavian vein could not be achieved in one-patient, and a conservative treatment was chosen. In our study, there is statistically significant difference regarding the multiplicity of lesions, at one-year follow-up, $77 \%$ of single successful lesions were patent while only $23 \%$ of multiple lesions were patent. This was in lines with Atalla et al. ${ }^{(2)}$, whose study showed that one-year patency rate of cases with single lesion was $91.6 \%$ and for those with multiple lesions was $8.3 \%$. There was a statistically significant difference between the patency rates between the two groups. One-year follow-up of previously successful cases showed that relation of recurrence to type of the lesion, there is statistically significant difference regarding the type of lesions as $56 \%$ of recurrent cases were occlusion in nature. This is in contrast to Atalla et al.(2), who documented that $80 \%$ of recurrent cases were stenotic. There were some limitations in this study. The small number of patients included in this study, mixed patients including, those with and without a history of catheter indwelling. Poor general conditions of the patients and funding issues were the main limitation regarding intervention in patients.

\section{Conclusion}

The percutaneous transluminal angioplasty with or without stenting according to our results is successful, safe, and effective technique in managing of central venous lesions in compromised AVF in hemodialysis patients. 
Financial support and sponsorship: None

Conflicts of interest: None

Acknowledgement: Authors would like to express their thankfulness and gratitude to Professor Amr MOGHAZY for reviewing as well as linguistic and scientific editing of this work.

\section{References}

1- Agarwal A. (2015) 'Endovascular interventions for central vein stenosis', krcp. Elsevier; -34(4), pp. 228-232.

2- Atalla A, Ahmed, $M$, and Taha $M$. (2020) 'Treatment of hemodialysis related-central venous stenosis: 1-year results of venoplasty and follow-up in 50 patients', ejs. Medknow Publications; 39(1), pp. 105.

3- Dammers R, Haan N, Planken N, and Vander-Sande M. (2003) 'Central vein obstruction in hemodialysis patients: results of radiological and surgical intervention', esvs. Elsevier; 26(3), pp. 317-321.

4- Hall K, and Farr B. (2004) 'Diagnosis and management of long-term central venous catheter infections', jvir. Elsevier; 15(4), pp. 327-334.

5- Ayarragaray J. (2003) 'Surgical treatment of hemodialysis-related central venous stenosis or occlusion: another option to maintain vascular access', jvs. Elsevier; 37(5), pp. 1043-1046.

6- Kim Y, Won J, Choi S, et al. (2009) 'Percutaneous treatment of central venous stenosis in hemodialysis patients: long-term outcomes', cirse. Springer; 32(2), pp. 271-278.

7- Quaretti P, Galli F, Moramarco L, et al. (2016) 'Stent grafts provided superior primary patency for central venous stenosis treatment in comparison with angioplasty and bare metal stent: a retrospective single center study on 70 hemodialysis patients', ves. SAGE Publications; 50(4), pp. 221230.

8- Sprouse L, Christopher J, Meier H, Demasi J, Gayle G, and Glickman H. (2004) 'Percutaneous treatment of symptomatic central venous stenosis angioplasty', jvs. Elsevier; 39(3), pp. 578-582.

9- Bakken M, Clinton D, Wael E, David E, Waldman L, and Davies G. (2007) 'Long-term outcomes of primary angioplasty and primary stenting of central venous stenosis in hemodialysis patients', jvs. Elsevier; 45(4), pp. 776783.

10-Surowiec M, Fegley J, Tanski J, et al. (2004) 'Endovascular management of central venous stenoses in the hemodialysis patient: results of percutaneous therapy', ves. Westminster Publications; 38(4), pp. 349-354.

11- Drewett, S. (2000) 'Complications of central venous catheters: nursing care', bjn. MA Healthcare London; 9(8), pp. 466-478.

12- Crowley A, Anna L, Gail E, et al. (2008) 'Venous thrombosis in patients with short-and long-term central venous catheter-associated Staphylococcus aureus bacteremia', ccm; 36(2), pp. 385-390.

13-Shi, Y, Meng Y, Liang W, et al. (2013) 'Endovascular treatment of central venous stenosis and obstruction in hemodialysis patients', cmj; 126(3), pp. 426-430.

14-Yadav M, Mukesh K, Madhurima L, et al. (2015) 'Endovascular treatment of central venous obstruction as a complication of prolonged hemodialysisPreliminary experience in a tertiary care center', ijri. Wolters -Medknow Publications; 25(4), pp. 368.

15- Oguzkurt L, Fahri T, Dilek T, et al. (2004) 'Impact of short-term hemodialysis catheters on the central veins: a catheter venographic study', ejr. Elsevier; 52(3), pp. 293-299. 
16- Nael K, Kee S, Houman S., and Katz G. (2009) 'Endovascular management of central thoracic veno-occlusive diseases in hemodialysis patients: a single institutional experience in 69 consecutive patients', jvir. Elsevier; 20(1), pp. 46-51.

17- Christidou P, Kalpakidis I, Kostas D, et al. (2006) 'Percutaneous transluminal angioplasty (PTA) and venous stenting in hemodialysis patients with vascular access-related venous stenosis or occlusion', ejr; 12(2), pp. 127-133.

18-Kalman P, Lindsay F, Sniderman W, and Vanderburgh L. (1998) 'Management of Upper Extremity Central Venous Obstruction Using Interventional Radiology', avs; 12(3), pp. 202-206.

19-Young $E$, Dawn $M$, Dykstra $A$, et al. (2002) 'Hemodialysis vascular access preferences and outcomes in the Dialysis Outcomes and Practice Patterns Study (DOPPS)', kjr. Elsevier; 61(6), pp. 2266-2271.

20- Aytekin C, Boyvata $F$, Yağmurdur $M$, Gökhan M, and Haberal M. (2004) 'Endovascular stent placement in the treatment of upper extremity central venous obstruction in hemodialysis patients', ejr. Elsevier; 49(1), pp. 8185. 